\title{
Dynamic Construction of Underground Mining Operations with Visualization in Virtual Reality
}

\author{
Oksana Ivina $^{1 *}$, Anastasia Belova ${ }^{1,2}$, Andrey $\mathrm{Klimchuk}^{2}$, and Tlek Akhmetgalym ${ }^{3}$ \\ ${ }^{1}$ T.F. Gorbachev Kuzbass State Technical University, 650000, Kemerovo, 28 Vesennya st., Russian \\ Federation \\ ${ }^{2} \mathrm{OOO}$ «SoftCATEN», 650010, Kemerovo, 34 Profsoyuznaya st., 34, Russian Federation \\ ${ }^{3}$ Kazakh Humanitarian Juridical Innovative University, EKR, Semey, 11Mangilik st., Republic of \\ Kazakstan
}

\begin{abstract}
Mining is one of the fastest growing solid mining industries worldwide. The intensification of production processes and increased economic efficiency by reducing costs and increasing production volumes, enhanced the intensive development in the use of modern, innovative technologies. Mixed reality technologies with the emphasis on creating the most natural interface, which could not be realized for a long time, are successfully being introduced into the production processes of the mining industry.The company SoftCATEN has developed a software package for the dynamic construction of underground mine workings with visualization in virtual reality. The principles of project implementation based on Unreal Engine 4 are developed. The proposed technology for constructing a coal mine model provides the conditions for a high-quality change in approaches to training processes and provides mining enterprises with tools for a full analysis of possible options for the development of mining operations.
\end{abstract}

\section{Introduction}

Innovative technologies and software are widely used to improve the quality and safety of work in production. Due to their flexibility, computer methods allow choosing effective tools for optimizing work in the coal industry, including at the stage of specialist training. The structure of the deposit visualized in space allows you to create and interpret geological sections, well profiles, tectonics, and also use the results in documenting the geological events of coal mines. The three-dimensional model of the deposit allows us to make the right assumptions and develop documentation in the future with suitable safe and effective solutions. It enables evaluation of virtual prototypes in a realistic form in the early stages of design and production. It also gives the opportunity to correct identified shortcomings already at the beginning of work, respectively, the costs associated with making changes in the design process are reduced. The perspective directions that have received the development of virtualization technologies are represented by visualization of the processes of modeling mining and geological information systems, the introduction of scheduling processes and design of industrial facilities, training of mining workers. Learning outside the immediate work environment provides only limited opportunities in real life and may not have a significant impact in the most stressful work environment. Learning tools based on virtual

\footnotetext{
*Corresponding author: k8enya@gmail.com
} 
reality can, on the contrary, provide a simulated impact of real working conditions without the related risks. To visualize a coal enterprise, the program used was the "3D Mine" software package developed by SoftCATEN, which allows constructing a volumetric production model based on graphic documentation of coal mines [1].

The authors [2] proposed visualization of the model in three main stages: visualization of the geology of the field; visualization of the existing mine network; visualization of design workings. To create a geological model of the field, exploration data is used, which are presented in a digital information database. Based on this information, a network of exploratory wells and test results are visualized. Visualization of geological disturbances in the form of surfaces is also possible. Building a model of the earth's surface and coal seams consists in digitizing contours or in indicating surface points with known coordinates. A three-dimensional surface model is formed by triangulating a network of points. First of all, he developed, close and balance coal seams are fixed in the model. Visualization of resources for a complete model of perception is marked by a variety of colors. The mine network corresponds to the mine plan.

A digital mine is presented, which represents a complex of a database and a model database in a single mine data management system, including five subsystems, including a data acquisition system, an integrated dispatch system, an applied engineering system, a data processing system, and a data management system. The product is a digital $3 \mathrm{D}$ visualized representation of the spatial information infrastructure of a real mine. The digital mine has 3 main functions - a data repository, an information directory and a digital platform. The developers also presented the modern development of the digital mine in the mining industry, scientific research and education. Examples of the modern construction of digital mines in China were given. Development trends and modern construction technologies at the digital mine were also presented [3].

The construction of a three-dimensional virtual scene of a coal mine is an objective requirement for the modernization and processing of information on coal mining, and also is a key technology in creating a "digital mine". Current world research, software and hardware, applied methods are aimed at studying 3D-geo-visualization of the mine surface and mine road. A methodology [4] has been developed for quick modeling of a large-scale virtual scene based on Arc / Info and Site-Builder3D with the implementation of automatic generation of 3D scenes from a 2D scene. A method has been implemented for converting mine roadway systems from 2D to 3D for the Dazhuang mine. Some applied issues were studied, including attribute request, coordinate request, distance measurement, collision detection, and dynamic interaction between $2 \mathrm{D}$ and $3 \mathrm{D}$ virtual scenes in the virtual scene of a mine surface plant and mine road. A prototype system was designed and implemented.

The application of a state and information monitoring system for servicing a complex, spatial distributed system of machines is considered, namely, a transport network of belt conveyors consisting of hundreds of drive units located in the mine area. The number of objects, their spatial location, the specific structure of the mining company, harsh environment, a variety of machines, etc. make this problem really difficult. Obviously, it is necessary to use specialized equipment, software, but, first of all, a set of procedures for collecting, checking, processing, storing, visualizing, making decisions, reporting, i.e. managing maintenance. All of these steps were combined and implemented as service management software called Diag Manager (CMMS class) $[5,6]$.

The progress of information technology allows you to convert geological phenomena into digital form. The possibilities of three-dimensional representation of geological formations in underground workings at a hard coal deposit in the southern part of the Upper Silesian coal basin in Central Europe, in Poland and the Czech Republic using the AutoCAD program are shown. Three-dimensional visualization of the results of mapping underground workings is presented and illustrated on two open workings. The selection criteria for these workings 
were based on their length and complexity of the geological conditions observed during their implementation. The described method can be used for spatial visualization of geological structures observed in other deposits, mines and existing workings. The presented methodology describes the task of visualizing underground mine workings in a typical geological aspect, considering the detailed visualization of geological installations identified on the side walls of mine workings [7,8].

The high prevalence of accidents and fatalities in the South African mining industry is often attributed to inefficient training methods and materials. The use of virtual reality technologies has been proposed as an alternative to existing approaches in training. VRtechnologies have already proved themselves in many industries as an effective method of training for various activities in the workplace, including in hazardous industries. For example, Division of Mining Technology successfully applies VR techniques for use as a training platform in the South African gold mining industry. Implemented virtual reality technologies received positive recommendations for creating effective training systems, including in the mining industry of South Africa [9].

To obtain the skills of various technological procedures, behavior in the case of nonstandard and emergency situations at production facilities of the mining industry, the task of training personnel is essential. In practice, such training is most often done on the basis of simulators and simulators that imitate reality. There are solutions designed for training or modeling a variety of situations, based on the interactive interaction of the user and the hardware-software system of modeling. There is a virtual mine type training complex on the market developed on the Microsoft Kinect platform. Libraries of data input, gesture processing and visualization were used, technology for interaction with the Kinect device was developed. In the future, it is supposed to continue the development of system modules for integration with the topology of real mines and complicated simulation scenarios [10]. Mixed reality is the result of combining real space and virtual holograms. One of the devices that allow broadcasting holographic objects of a project carried out in the Micromine mining and geological environment is Microsoft's HoloLens glasses, which complement threedimensional holograms with a user's presence in the real world, tied to real-world objects (building models, reference information, video clips). In the obtained interface of interaction between a person and a virtual environment, the display of objects is similar to real objects. The objective of the work was the implementation of the dynamic construction of a virtual mine and logical interaction with equipment using the capabilities of hybrid reality to create a software environment for training personnel and university students before going down to the mine.

\section{Results and discussion}

As a result, a system for the dynamic construction of a virtual mine model using virtual reality technology was developed, the mine, its underground part, mine workings and installed technical equipment were visualized. More than 30 3D objects were imposed, such as the KRUW cell, the KTPVSh-630 transformer, the PVI-250 starter, roadheaders and shearers, roof supports, conveyors, anchors and other equipment, and elements of the mining industry. Data for the construction of virtual mines are taken from the database of the 3D Mine program.

Using the module «3D Mine" as a part of software package allows you to improve the security of the mines by creating an integrated information system for collection, processing and analysis of information to take timely and effective management decisions, and also carry out preventive measures, and in emergency situations, quickly implement the measures of emergency response plans. It allows you to solve the problems of optimizing the work schedule and the schedule of scheduled preventive measures, which reduces production costs. 
The module is a multi-user application with a distributed database installed on the workplaces of employees of structural divisions involved in work processes. The 3D Mine program module provides simultaneous operation of all users in real time with separation of access rights to data depending on work responsibilities. It is represented by a database, software that collects data from related subsystems, software that performs the function of exchanging data with other control levels, and application software running on the workstation [11-13].

The coordinates of the junctions of the workings and the location of the objects in the mine are provided. Mines in virtual reality are built on the basis of real data from paved workings underground, due to which the virtual mine is as close as possible to the real one, Fig. 1.

At the next stage, the "VR Mine" program is connected to the database (DB) using a plugin written by SoftCATEN. After that, a request is sent to receive data from the database, identifiers of the mine with which the construction of the mine will begin are determined. Based on the data obtained, splines are constructed from the start node of the workings to the end node of the workings, after which a mesh of a certain type is laid on the spline, on which sockets are specified in a certain order.

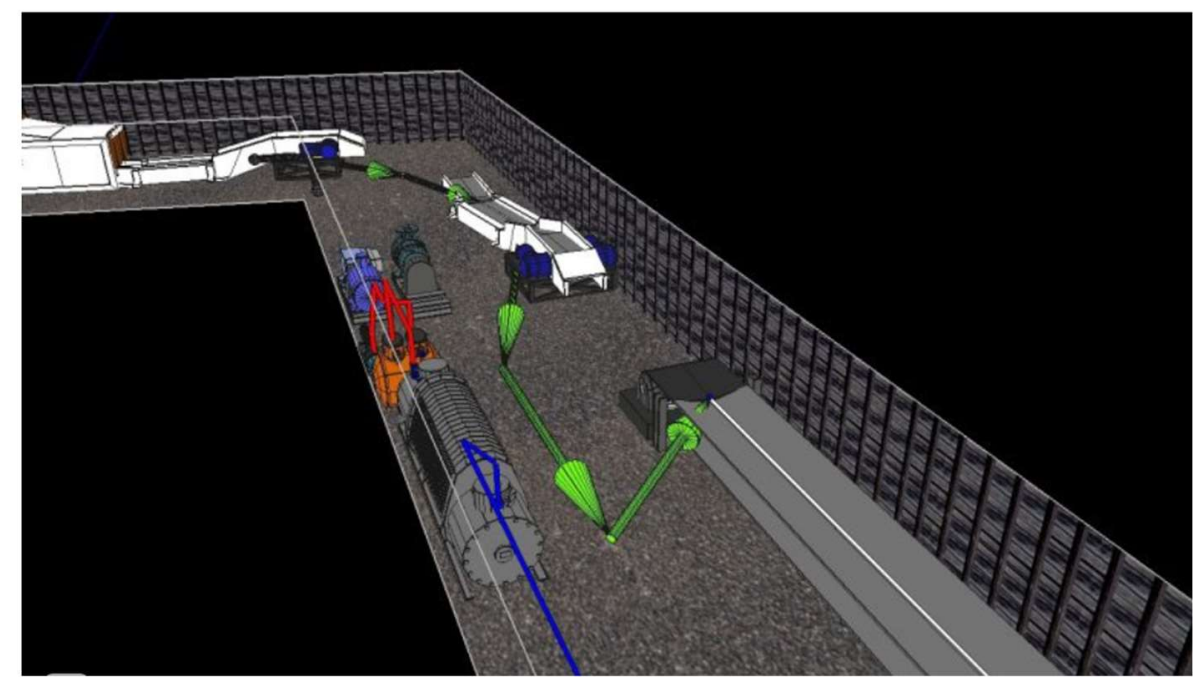

Fig. 1. An example of a three-dimensional model of technological equipment with the exact reference point in the mine workings in the program « $3 \mathrm{D}$ mine».

Between workings, nodes are built on the principle of procedural generation. To generate the corner between the workings, it is needed to take the coordinates of the socket and specify this point for the corner. For editing, all attachment points are described in corner maps. A corner map is a table based on a data structure. Each table describes the number of sides attached to the corner, i.e. if the corner does not connect the workings, but is the beginning or the end, then the map is for one side, if we have two workings, then the map is for two sides, etc.

Objects are built in the coordinates specified in the database. For objects to interact with each other, an object is needed from which the cable exits in order to transfer data about the object to which this cable is connected. Thus we can trace the order of connecting objects to each other. When the technical equipment is turned on at the constructed level, power is supplied to all objects connected to the switched on element. DataTables is a related data table where data fields can be any valid UObject property, including resource references. 
Object references are stored in the DataTable. An example of filling the table is shown in Fig. 2.

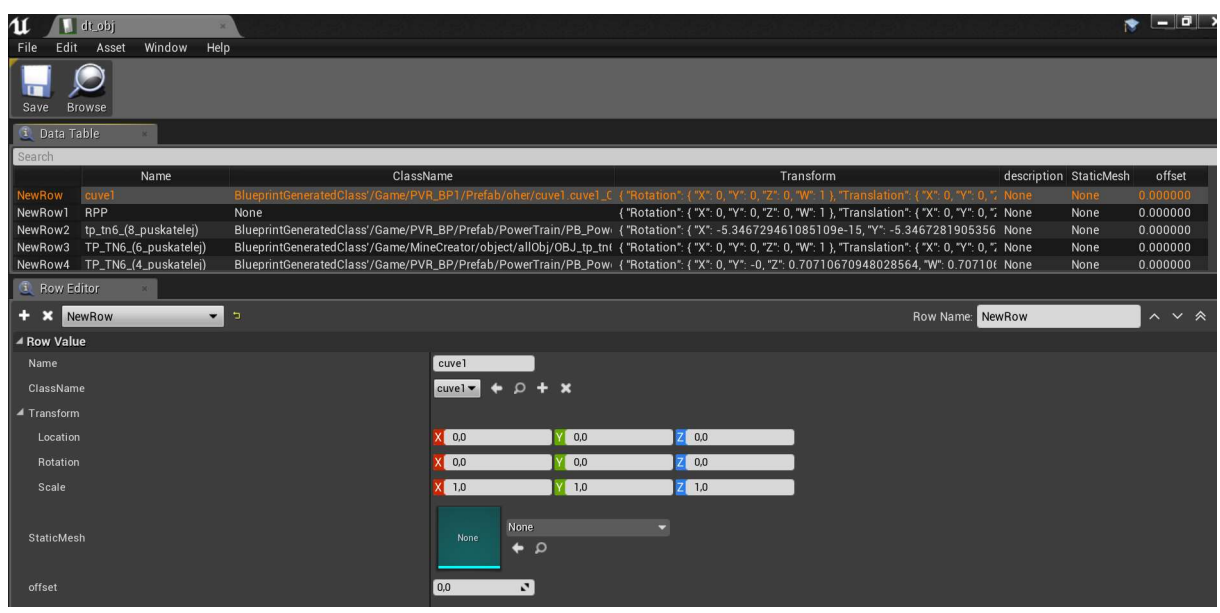

Fig. 2. Example filling DataTable.

Triggers are responsible for the dynamic construction of workings. Initially, workings are developed that have a common node with a working that has an initial identifier. Then, when building a mine, the triggers consisting of BoxCollision spawn as shown in Fig. 3.

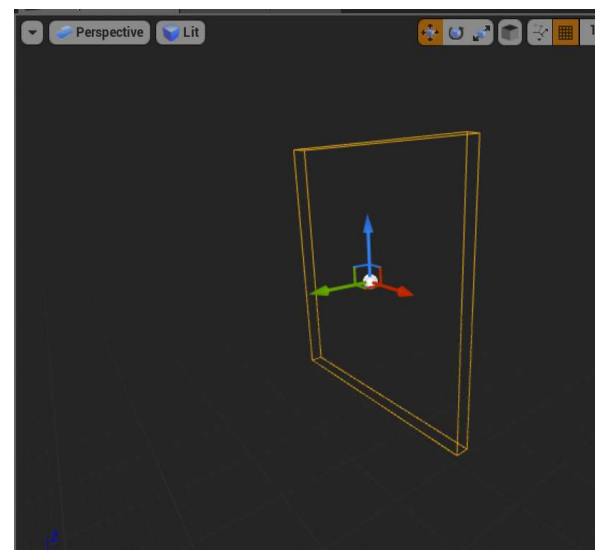

Fig. 3. A trigger.

During the development process, the BeginOverlap procedure was used, which is executed when the component collides with other objects. If a player crosses BoxCollision, the BeginOverlap event is triggered, where the actor who crossed BoxCollision is transferred, and the working in which the game character is located. After that, a check is made for the presence of neighboring workings from the one in which the game character is located. If there are neighboring workings, then the working in which player is located becomes a point for constructing neighboring workings according to the previously described algorithm. Fig. 4 shows an example of a top view of a constructed mine. 


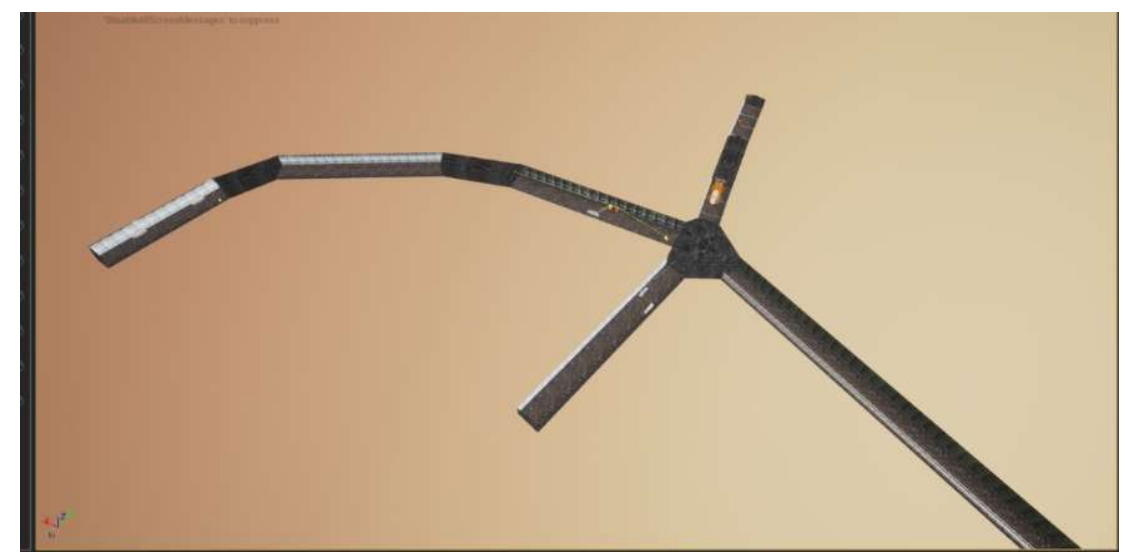

Fig. 4. Example of a top view of a mine.

Thus, the developed "VR Mine" module has tools for creating a database that allows realtime exchange with the "3D mine" module and provides the necessary and sufficient information for the operation of the virtual reality software core. The "VR Mine" module, which has an interface to work with the "3D mine" database, receives all the necessary information for constructing a virtual model of mine workings, as well as the location of equipment, communications, safety equipment, warning systems, etc. located in these workings. The necessary information is the actual coordinates of the location of objects, sizes, technical specifications.

\section{Conclusion}

The statistics of industrial accidents shows that the mining industry is one of the most dangerous. Therefore, there is a need for new solutions that will improve the process of professional adaptation and at the same time have a significant impact on improving the situation in the field of labor protection and safety, especially among the youngest workers. Using virtual reality technology (VR) allows you to acquire and practice the correct behavior of miners in a controlled and safe environment.

The "VR Mine" software module has been developed, which includes a set of components - a database, software that integrates with the created "3D mine" module, software for editing 3D models of objects, and a training script module. Over $303 \mathrm{D}$ objects of mining equipment and various elements of the mining industry have been imposed. The module includes a library of training scenarios that provides various training conditions and assesses the correctness of actions according to specified criteria. The training scenario involves the creation in virtual reality of unforeseen emergency situations that training participants must eliminate. The script also includes all the possible solutions of situations for students. The software module "VR Mine" allows the simultaneous work of three training participants. It should be noted that the development of such educational complexes will only accelerate, the tasks set for students will become more complicated, since this virtual reality technology becomes more accessible for consumers every year. It is assumed that such a threedimensional approach, in addition, will make it possible to predict geological and mountain hazards when planning safe and effective mining operations and streamline deposit management. 


\section{REFERENCES}

1. L. Wu, D. Che J, Coal Sci., 3, 415 (2008)

2. Z. Hao, W. Hao, G. Yang, Com. and Inf. Proc., 3, 206 (2012)

3. Y. Wang, Y. Fu, E. Fu, Geo Spat Inf Sci, 4, 287 (2007)

4. P. Stefaniak, R. Zimroz, R. Krol, J. Gorniak-Zimroz, W. Bartelmus, M. Hardygora, Condition Monitoring of Machinery in Non-Stationary Operations, 5, 497 (2012)

5. B. Zhou, J. Xu, S. Peng, J. Geng, F. Yan, Int. J. Rock Mech. Min. Sci., 122, 104083 (2019)

6. M. Marcisz, K. Probierz, M. Ostrowska-Łach, J. Sustain. Min., 1, 34 (2018)

7. T. Urych, M. Głogowska, R. Warzecha, A. Wątor, J. Chećko, SGEM, 2, 67 (2019)

8. E. A. van Wyk, M. R. de Villiers, J South Afr Inst Min Metall., 5, 427 (2019)

9. B. Lange, C. Y. Chang, E. Suma, B. Newman, A. S. Rizzo, M. Bolas, IEEE, 1, 7 (2011)

10. A. Stork, N. Sevilmis, D. Weber, D. Gorecky, C. Stahl, M. Loskyll, F. Michel, IEEE, 2, $11(2012)$

11. B. Lange, A. Rizzo, C. Y. Chang, E. A. Suma, M. Bolas, I/ITSEC, 1, 6 (2011)

12. V. R. Kamat, J. C. Martinez, J. Comput. Civ. Eng., 15(4), 329 (2001)

13. J. Li, Y. Liu, J. Xie, X. Wang, X. Ge, Appl. Sci., 10(3), 771 (2020) 